\title{
ONE-FIELD EQUATIONS FOR TWO-PHASE FLOWS
}

\author{
MURRAY RUDMAN 1
}

(Received 4 January 1996; revised 15 July 1996)

\begin{abstract}
A new derivation of the averaged heat and mass transport equations for two-phase flows is presented. A volume averaging technique is used in which averaging is performed over both phases simultaneously in order to derive equations that describe transport in the mixture, rather than transport in each phase. The derivation is particularly applicable to incompressible liquid/solid systems in which the two phases are tightly coupled. An example of the numerical solution of the equations is then presented in which a thermally convecting suspension is modelled. It is seen that large-scale instability can result from the interaction of thermal and compositional density gradients.
\end{abstract}

\section{Introduction}

The most common approach used to derive equations that describe transport in twophase flow systems is to define averaged properties by averaging over each phase separately. Equations for the transport of the averaged properties are then derived by averaging the micro-scale transport equations over each phase. Ensemble averaging [12], temporal averaging [10], spatial averaging [20] and a combination of spatial and temporal averaging [5] have all been used to derive transport equations. Averaging over each phase independently is termed here a two-field approach because each micro-scale quantity has two averaged fields associated with it, and a separate transport equation for each phase. The resulting equations describe transport in one phase only, and are coupled through interaction terms whose form must be postulated. A good exposition of this type of derivation is given in [4].

It is also possible io define mixture averaged variables and derive equations for the mixture. The 'mixture' approach is termed here the one-field approach and the resulting equations are termed the one-field equations. Its use is appropriate when the two fields are strongly coupled and mass, momentum and heat transport due to large-

${ }^{1}$ Commonwealth Scientific and Industrial Research Organisation, Division of Building, Construction and Engineering, PO Box 56, Highett, Victoria 3190, Australia

(C) Australian Mathematical Society, 1997, Serial-fee code 0334-2700/97 
scale motions of the mixture are more important than transfer due to interpenetration of the phases. Examples of physical processes that satisfy these requirements are the settling of crystals in magma chambers (see [16]) and the densifying bed of Bayerprocess gravity thickeners which may be undergoing active thermal convection.

In this paper, a two-phase fluid consisting of an incompressible, constant density, viscous liquid and a dilute dispersion of identical solid particles is considered. For simplicity, disordered turbulence arising both from interpenetration of the phases and from the large-scale motion of the mixture is not considered, although its inclusion is not ruled out by the one-field approach.

\section{Averaging}

When deriving averaged transport equations, the existence of three length scales is implicitly assumed:

- The dispersed-phase length scale over which there can be significant fluctuations in velocity, density and stress (characterised by the separation between particles).

- The averaging (control volume) length scale which is many times greater than the dispersed-phase length scale.

- The dynamic length scale which is many times greater than the averaging length scale. It is the scale over which significant variations in the macro-scale flow occurs.

All flow variables referring to the micro-scale or exact flow appear unbarred, for example, $\boldsymbol{U}, P, \rho$. The mixture-averaged value of $Q$ in a neighbourhood of $\boldsymbol{r}$ in both phases of the flow is denoted $\bar{Q}(r)$ and is defined using a mixture average as

$$
\bar{Q}(\boldsymbol{r})=\frac{1}{\mathscr{V}} \int_{C V} Q\left(\boldsymbol{r}+\boldsymbol{r}^{\prime}\right) d \boldsymbol{V}^{\prime},
$$

where $\mathscr{V}$ is the neighbourhood of $\boldsymbol{r}$ (or control volume, $\boldsymbol{C V}$ ) over which the integration takes place. It is uniform in time and space, and is

$$
\mathscr{V}=\int_{C V} d V^{\prime}
$$

Throughout this paper, the dispersed phase is subscripted or superscripted with a ' $d$ ' and the continuous liquid phase with a ' $c$ '. The phase function, $\beta_{m}(\boldsymbol{r})$, defines which material exists at position $\boldsymbol{r}$ and is defined for the $m^{t h}$ phase $(m=d$ or $c)$ as

$$
\beta_{m}(r)= \begin{cases}1 & \text { if } \boldsymbol{r} \text { lies in phase } m \\ 0 & \text { otherwise. }\end{cases}
$$


The spatial derivative of the phase function $\beta_{d}$ is (see [20])

$$
\nabla \beta_{d}(\boldsymbol{r})=-\delta\left(\boldsymbol{r}_{l}\right) \frac{\boldsymbol{r}}{|\boldsymbol{r}|}=-\delta\left(\boldsymbol{r}_{I}\right) \boldsymbol{n}_{d}
$$

where $\boldsymbol{n}_{d}$ is the unit normal to the interface directed away from the dispersed phase and $\delta\left(\boldsymbol{r}_{l}\right)$ is a delta function that defines the interface. Variables that refer to the micro-scale value of $Q$ only within phase $m$ are defined using

$$
Q_{m}=\beta_{m} Q
$$

with the following being true in general:

$$
Q \equiv \beta_{d} Q_{d}+\beta_{c} Q_{c}
$$

The void fraction of phase $m, \epsilon_{m}$, is the mixture-averaged phase function,

$$
\epsilon_{m}=\overline{\beta_{m}}
$$

The intrinsic phase average of $Q$ is the average of $Q$ over one phase of the mixture only. For the $m^{t h}$ phase it is

$$
\overline{Q(\boldsymbol{r})}^{m}=\frac{\int_{C V} \beta_{m}\left(\boldsymbol{r}+\boldsymbol{r}^{\prime}\right) Q\left(\boldsymbol{r}+\boldsymbol{r}^{\prime}\right) d \boldsymbol{V}^{\prime}}{\int_{C V} \beta_{m}\left(\boldsymbol{r}+\boldsymbol{r}^{\prime}\right) d V^{\prime}}=\frac{\overline{\beta_{m} Q_{m}}}{\epsilon_{m}} .
$$

Hence

$$
\overline{\beta_{m} Q_{m}}=\epsilon_{m} \bar{Q}^{m} .
$$

From equations (4) and (7),

$$
\bar{Q}=\epsilon_{d} \bar{Q}^{d}+\epsilon_{c} \bar{Q}^{c}
$$

The relationship between the average of a derivative and the derivative of an average can be seen from (1) to be

$$
\begin{aligned}
\nabla \bar{Q}(\boldsymbol{r}) & =\nabla\left(\frac{1}{\mathscr{V}} \int_{C V} Q\left(\boldsymbol{r}+\boldsymbol{r}^{\prime}\right) d \boldsymbol{V}^{\prime}\right) \\
& =\frac{1}{\mathscr{V}} \int_{C V} \nabla Q\left(\boldsymbol{r}+\boldsymbol{r}^{\prime}\right) d \boldsymbol{V}^{\prime} \\
& =\frac{\bar{\nabla} Q}{(\boldsymbol{r})} .
\end{aligned}
$$


The derivative may be taken into the integral in (9) because the limits of integration depend only on the primed coordinates. This result is valid even when $Q(\boldsymbol{r})$ is not continuously differentiable or even continuous within the control volume. (To see this, write $Q$ in (9) using (4) and expand the derivative, noting that $\beta_{c}+\beta_{d} \equiv 1$.)

Finally, relative variables are defined in terms of the averaged two-field variables as

$$
Q_{R}=\bar{Q}^{d}-\bar{Q}^{c}
$$

from which

$$
\bar{Q}^{d}=\bar{Q}+\epsilon_{c} Q_{R} \quad \text { and } \quad \bar{Q}^{c}=\bar{Q}-\epsilon_{d} Q_{R} .
$$

Decomposition of a micro-scale variable into a sum of its mean and fluctuating parts is used frequently below and can be accomplished by defining the mean part to be the mixture average in which case

$$
Q=\bar{Q}+Q^{*},
$$

where (for example) $U^{*}$ represents the deviation of $\boldsymbol{U}$ from $\bar{U}$ due to the relative motion of the two components of the mixture. This is in contrast to the Reynolds decomposition used in turbulence modelling in which $U^{*}$ represents the deviation of the velocity field from the mean as a result of disordered turbulent fluctuations in the fluid. Decomposition of a variable can also be undertaken by defining the mean part to be an intrinsic phase average,

$$
Q_{m}=\bar{Q}^{m}+Q_{m}^{\prime \prime}
$$

To the order of approximation that is used throughout the following derivations, $\overline{\bar{Q}}=\bar{Q}$ and ${\overline{Q^{m}}}^{m}=\bar{Q}^{m}$, thus ${\overline{Q^{*}}}^{m}=0$ and ${\overline{Q_{m}^{\prime \prime}}}^{m}=0$. To see the relationship between the two constructions (12) and (13) consider the correlation $\overline{(P Q)}$. Equation (12) gives

$$
\overline{(P Q)}=\bar{P} \bar{Q}+\overline{P^{*} Q^{*}}
$$

and (13) gives

$$
\begin{aligned}
& \overline{P Q}=\epsilon_{d}\left(\bar{P}^{d} \bar{Q}^{d}+{\overline{P_{d}^{\prime \prime} Q_{d}^{\prime \prime}}}^{d}\right)+\epsilon_{c}\left(\bar{P}^{c} \bar{Q}^{c}+{\overline{P_{c}^{\prime \prime} Q_{c}^{\prime \prime}}}^{c}\right) \\
& =\bar{P} \bar{Q}+\epsilon_{d} \epsilon_{c} P_{R} Q_{R}+\epsilon_{d}{\overline{P_{d}^{\prime \prime} Q_{d}^{\prime \prime}}}^{d}+\epsilon_{c}{\overline{P_{c}^{\prime \prime} Q_{c}^{\prime \prime}}}^{c} \text {. }
\end{aligned}
$$

The terms $\epsilon_{d} \overline{P_{d}^{\prime \prime} Q_{d}^{\prime \prime}}$ and $\epsilon_{c} \overline{P_{c}^{\prime \prime} Q_{c}^{\prime \prime}}$ are the two-phase equivalent to the Reynolds stresses and are assumed to be negligible in the absence of disordered turbulence. Thus (15) becomes

$$
\overline{P Q} \approx \bar{P} \bar{Q}+\epsilon_{d} \epsilon_{c} P_{R} Q_{R}
$$


from which it is seen that

$$
\overline{P^{*} Q^{*}} \approx \epsilon_{d} \epsilon_{c} P_{R} Q_{R}
$$

\section{Equations of mass and momentum transport}

3.1. Continuity. At any point in the flow the micro-scale equation of mass conservation is

$$
\frac{\partial \rho}{\partial t}+\nabla \cdot(\rho \boldsymbol{U})=0,
$$

and incompressibility is

$$
\nabla \cdot \boldsymbol{U}=0
$$

Averaging (18) results in

$$
\frac{\partial \bar{\rho}}{\partial t}+\nabla \cdot\left(\bar{\rho} \overline{\boldsymbol{U}}+\epsilon_{d} \epsilon_{c} \delta \rho \boldsymbol{U}_{R}\right)=0,
$$

where $\delta \rho=\rho_{d}-\rho_{c}$. Clearly

$$
\nabla \cdot \bar{U}=0,
$$

which states that the mixture as a whole is incompressible. Neither the divergence of $\overline{\boldsymbol{U}}^{d}$ nor $\overline{\boldsymbol{U}}^{c}$ is necessarily zero.

3.2. Momentum equation. In the absence of interfacial forces, the momentum equation at any point in the mixture is

$$
\frac{\partial}{\partial t}(\rho \boldsymbol{U})+\nabla \cdot(\rho \boldsymbol{U} \boldsymbol{U})=\rho \boldsymbol{g}+\nabla \cdot \sigma,
$$

where $g$ is gravitational acceleration and $\sigma$ is the stress tensor, equal (for a Newtonian fluid) to

$$
\sigma=-P \boldsymbol{I}+\mu\left[\nabla \boldsymbol{U}+\nabla \boldsymbol{U}^{T}\right] .
$$

Averaging (22) yields (using equations (4), (7) and (9))

$$
\frac{\partial}{\partial t}(\bar{\rho} \widetilde{\boldsymbol{U}})+\nabla \cdot\left(\epsilon_{d} \rho_{d} \overline{\boldsymbol{U U}}^{d}+\epsilon_{c} \rho_{c} \overline{\boldsymbol{U U}}^{c}\right)=\bar{\rho} \boldsymbol{g}+\nabla \cdot \bar{\sigma},
$$

where the mass-weighted velocity $\widetilde{\boldsymbol{U}}$ is defined using $\bar{\rho} \widetilde{\boldsymbol{U}}=\epsilon_{d} \rho_{d} \overline{\boldsymbol{U}}^{d}+\epsilon_{c} \rho_{c} \overline{\boldsymbol{U}}^{c}$. Using (11) and (13) and neglecting the two-phase Reynolds stresses $\left({\overline{U_{d}^{\prime \prime} U_{d}^{\prime \prime}}}^{m}\right)$ allows the mixture-averaged momentum equation to be written as

$$
\frac{\partial}{\partial t}(\bar{\rho} \widetilde{\boldsymbol{U}})+\nabla \cdot\left(\bar{\rho} \overline{\boldsymbol{U}} \overline{\boldsymbol{U}}+\delta \rho \epsilon_{\mathrm{d}} \epsilon_{\mathrm{c}}\left(\overline{\boldsymbol{U}} \boldsymbol{U}_{R}+\boldsymbol{U}_{R} \overline{\boldsymbol{U}}\right)+\bar{\rho} \epsilon_{d} \epsilon_{\mathrm{c}} \boldsymbol{U}_{R} \boldsymbol{U}_{R}\right)=\bar{\rho} \boldsymbol{g}+\nabla \cdot \bar{\sigma}
$$


As in two-field modelling the stress term must be modelled by postulating a constitutive equation. Constitutive modelling is the most problematic aspect of deriving equations for multi-phase flow, and is accomplished here by first splitting the total stress into pressure and viscous components,

$$
\nabla \cdot \bar{\sigma}=-\nabla \bar{P}+\nabla \cdot \bar{\tau}
$$

In the case of suspension flow, the results of [14] show that the gradient of the phaseaveraged pressure in the dispersed phase is equal to the gradient of the phase-averaged pressure in the continuous phase, that is,

$$
\nabla \bar{P}^{d}=\nabla \bar{P}^{c} .
$$

This result is physically reasonable and states that any excess internal pressure within a particle or droplet due to surface tension has no relevance to the pressure forces acting on the particle's exterior and hence no relevance to its translational motion through the fluid. The approach taken here is to define a mixture-averaged pressure, $\bar{P}$, the gradient of which acts equally on both the dispersed and continuous phases,

$$
\nabla \bar{P}=\nabla \bar{P}^{d}=\nabla \bar{P}^{c}
$$

A suitable constitutive relationship for the mixture-averaged viscous stress $\bar{\tau}$ can be postulated by considering a suspension of small liquid droplets. Taylor [17] derives an expression for the effective viscosity of the mixture as

$$
\mu_{T}=\mu_{c}\left(1+\frac{5}{2} \epsilon_{d} \frac{\mu_{d}+\frac{2}{5} \mu_{c}}{\mu_{d}+\mu_{c}}\right) .
$$

As $\mu_{d} \rightarrow \infty, \mu_{T} \rightarrow \mu_{E}$ where $\mu_{E}$ is given by the Einstein formula [7] for a suspension of solid spheres, that is,

$$
\mu_{E}=\left(1+2.5 \epsilon_{d}\right) \mu_{c} .
$$

The effective viscosity defined in (27) is consistent with the results presented in [9] in which the total viscous stress in a solid suspension is shown to be equal to $\left(1+2.5 \epsilon_{d}\right) \mu_{c} \bar{E}_{i j}$, where $\bar{E}_{i j}$ is twice a suitably defined average rate of strain tensor. In [9] it is stated that $\bar{E}_{i j}$ can be written using either $\overline{\boldsymbol{U}}^{c}$ or $\widetilde{\boldsymbol{U}}$. Based on the derivation of $\mu_{T}$ in [17] it could be argued that the appropriate rate of strain tensor for which $\mu_{E}$ applies should be defined using $\bar{U}$. This is, for example, the rate of strain that would be used when deriving suspension viscosity from stress measurements in a rheometer. Thus, the constitutive model chosen here for $\bar{E}_{i j}$ is

$$
\bar{E}_{i j}=\left(\frac{\partial \bar{U}_{i}}{\partial x_{j}}+\frac{\partial \bar{U}_{j}}{\partial x_{i}}\right) .
$$


This model not only has a physical basis, but has the added advantage that it facilitates numerical solution of the equations. When the two-field equations are derived from the one-field equations (see section 4), this constitutive model gives rise to two-field equations that do not satisfy the hypothesis of phase separation suggested in [5]. Nevertheless, the importance of this hypothesis in deriving constitutive relationships has not been generally shown, and this shortcoming is not believed to invalidate the use of (28). The total average mixture stress is thus written

$$
\nabla \cdot \bar{\sigma}=-\nabla \bar{P}+\nabla \cdot\left(\mu_{E}\left[\nabla \bar{U}+\nabla \bar{U}^{T}\right]\right) .
$$

The range of validity of the Einstein formula (27) can be extended using empirical relationships [18].

3.3. Mixture-averaged velocity. Density can be removed from (22) using (18), giving

$$
\frac{\partial \boldsymbol{U}}{\partial t}+\nabla \cdot(\boldsymbol{U} \boldsymbol{U})=\boldsymbol{g}+\frac{1}{\rho} \nabla \cdot \sigma .
$$

Averaging this equation and using (16) yields

$$
\frac{\partial \bar{U}}{\partial t}+\nabla \cdot\left(\bar{U} \bar{U}+\epsilon_{d} \epsilon_{c} \boldsymbol{U}_{R} \boldsymbol{U}_{R}\right)=\boldsymbol{g}+\overline{\left(\frac{1}{\rho} \nabla \cdot \sigma\right)} .
$$

The only unknown quantity in (31) is the stress term, which is the average of a product of the stress divergence and inverse density. It is in the closure of this term where the fundamental difference between one- and two-field derivations arises. The derivation below is both involved and approximate, but is necessary to show how the closure is made. As in the previous section, it is initially assumed that the dispersed phase consists of droplets of a second fluid (with particle suspension flow resulting as $\left.\mu_{d} \rightarrow \infty\right)$.

The stress term in (31) can be written

$$
\overline{\left(\frac{1}{\rho} \nabla \cdot \sigma\right)}=\frac{1}{\mathscr{V}}\left[\int_{\mathscr{Y}_{d}}\left(\frac{1}{\rho_{d}} \nabla \cdot \sigma^{d}\right) d \boldsymbol{V}^{\prime}+\int_{\mathscr{Y}_{c}}\left(\frac{1}{\rho_{c}} \nabla \cdot \sigma^{c}\right) d \boldsymbol{V}^{\prime}\right],
$$

where $\mathscr{V}_{d}$ and $\mathscr{V}_{c}$ are those parts of $C V$ in phases $d$ and $c$ respectively. Nomenclature describing the control volume is now required (see Figure 1). The surface of the control volume, $C V S$, cuts through both phases - $C V S_{d}$ is that part of $C V S$ lying in the dispersed phase and $C V S_{c}$ is that part lying in the continuous phase. The surface of a single droplet lying entirely in $C V$ is $S_{p}$ and the sum of all such droplet surfaces is $\Sigma S_{p}$. For a droplet cut by the control volume surface, that portion of the droplet's 


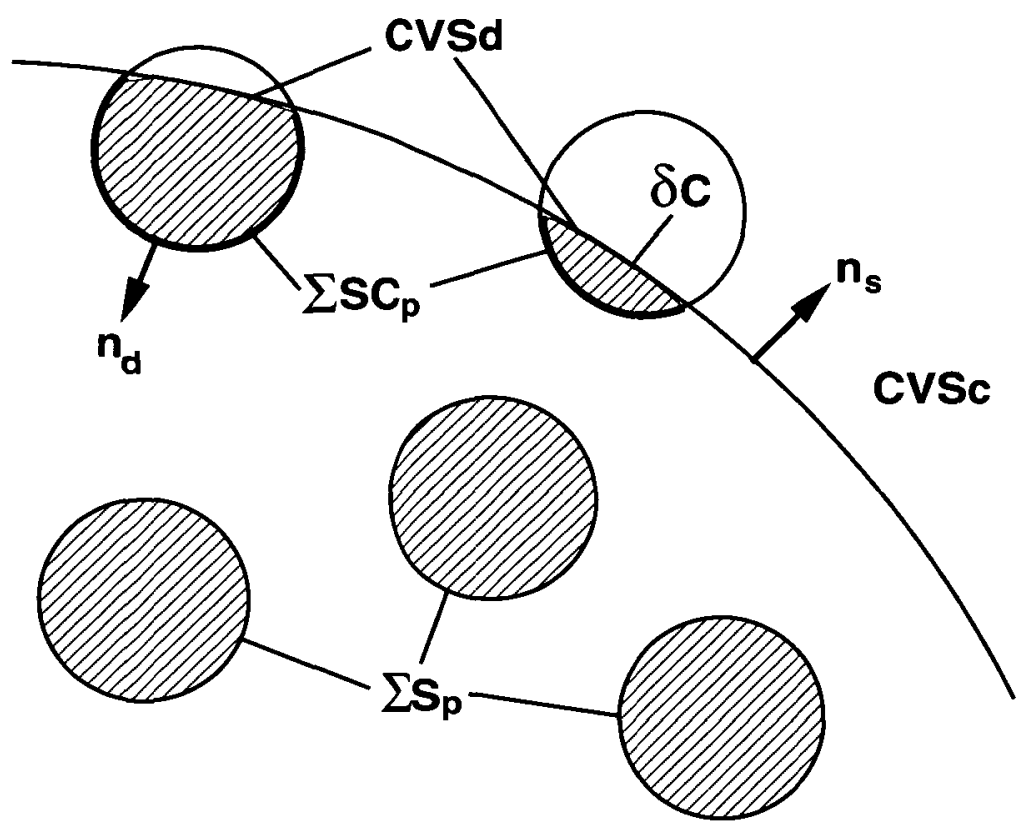

FIGURE 1. The control volume.

surface lying in $\boldsymbol{C V}$ is $S_{C_{n}}$ and $\Sigma S_{C_{p}}$ is the sum of all such surfaces. The part of the control volume surface interior to a droplet is denoted by $\delta C$, and $C V S_{d}$ is the sum of such contributions from all cut droplets. The outward normals from each phase are $\boldsymbol{n}_{d}$ and $\boldsymbol{n}_{c}$. The outward normal to the control volume is $\boldsymbol{n}_{s}$.

Because $\rho_{d}$ and $\rho_{c}$ are constant they can be removed from the integrals in (32). Using Gauss' theorem and splitting each of the resulting surface integrals into an integral over the interface plus an integral over either $C V S_{d}$ or $C V S_{c}$ yields

$$
\begin{aligned}
\overline{\left(\frac{1}{\rho} \nabla \cdot \sigma\right)}=\frac{1}{\mathscr{V}} & {\left[\left(\frac{1}{\rho_{d}}\right) \int_{C V S d} \sigma^{d} \cdot \boldsymbol{n}_{s} d \boldsymbol{S}^{\prime}+\left(\frac{1}{\rho_{c}}\right) \int_{C V S c} \sigma^{c} \cdot \boldsymbol{n}_{s} d \boldsymbol{S}^{\prime}\right] } \\
& -\frac{1}{\mathscr{V}}\left[\left(\frac{1}{\rho_{c}}-\frac{1}{\rho_{d}}\right) \int_{\Sigma S_{p}+\Sigma S_{C_{p}}} \sigma^{c} \cdot \boldsymbol{n}_{c} d \boldsymbol{S}^{\prime}\right] .
\end{aligned}
$$

It may be shown [8] that

$$
\frac{1}{\mathscr{V}} \int_{C V S m} Q_{m} \boldsymbol{n}_{s} d \boldsymbol{S}^{\prime}=\nabla\left(\epsilon_{m} \bar{Q}^{m}\right) .
$$


Using (34) in the first two integrals of (33), manipulating the third by changing the direction of the surface normal and using the decomposition (13) of $\sigma^{c}$ gives

$$
\begin{aligned}
\overline{\left(\frac{1}{\rho} \nabla \cdot \sigma\right)}= & \frac{1}{\rho_{d}} \nabla \cdot\left(\epsilon_{d} \bar{\sigma}^{d}\right)+\frac{1}{\rho_{c}} \nabla \cdot\left(\epsilon_{c} \bar{\sigma}^{c}\right) \\
& +\frac{1}{\mathscr{V}}\left(\frac{1}{\rho_{d}}-\frac{1}{\rho_{c}}\right)\left[\int_{\Sigma S_{p}+\Sigma S_{c_{p}}} \bar{\sigma}^{c} \cdot \boldsymbol{n}_{d} d \boldsymbol{S}^{\prime}+\int_{\Sigma S_{p}+\Sigma S_{c_{p}}} \sigma_{c}^{\prime \prime} \cdot \boldsymbol{n}_{d} d \boldsymbol{S}^{\prime}\right]
\end{aligned}
$$

The approach of [14] can be extended to show [15] that in the absence of surface tension

$$
\bar{\sigma}^{d}=\bar{\sigma}^{c}(=\bar{\sigma})
$$

That is, the total phase-averaged stresses in the two phases are equal. Inclusion of surface tension would result in an additional component to the dispersed-phase stress that would exactly balance the surface tension contribution, and a modified version of (36) would still hold. Because the inclusion of surface tension complicates the derivation considerably (at the same time as yielding the same end result for dilute suspension flows), it is neglected in all subsequent discussion. Equation (36) allows the first two terms on the right-hand side of (35) to be written

$$
\frac{1}{\rho_{d}} \nabla \cdot\left(\epsilon_{d} \bar{\sigma}^{d}\right)+\frac{1}{\rho_{c}} \nabla \cdot\left(\epsilon_{c} \bar{\sigma}^{c}\right)=\left(\frac{\epsilon_{d}}{\rho_{d}}+\frac{\epsilon_{c}}{\rho_{c}}\right) \nabla \cdot \bar{\sigma}+\left(\frac{1}{\rho_{d}}-\frac{1}{\rho_{c}}\right) \bar{\sigma} \cdot \nabla \epsilon_{d} .
$$

The integral of $\bar{\sigma}^{c}$ over the interface in the third term on the right-hand side of (35) is manipulated by adding and subtracting an integral over $C V S_{d}$, and expanding the first integral in a Taylor series about the control volume centre to give

$$
\begin{aligned}
& \frac{1}{\mathscr{V}} \int_{\Sigma S_{p}+\Sigma S_{C_{p}}} \bar{\sigma}^{c} \cdot \boldsymbol{n}_{d} d \boldsymbol{S}^{\prime} \\
& =\frac{1}{\mathscr{V}} \int_{\substack{\Sigma S_{p}+\Sigma S_{C_{p}} \\
+C V S_{d}}} \bar{\sigma}^{c} \cdot \boldsymbol{n}_{d} d \boldsymbol{S}^{\prime}-\frac{1}{\mathscr{V}} \int_{C V S d} \bar{\sigma}^{c} \cdot \boldsymbol{n}_{s} d \boldsymbol{S}^{\prime} \\
& =\frac{1}{\mathscr{V}} \int_{\substack{\Sigma S_{p}+\Sigma S_{C_{p_{p}}} \\
+C V S_{d}}}\left[\bar{\sigma}^{c}(\boldsymbol{r})+\left(\boldsymbol{r}^{\prime}-\boldsymbol{r}\right) \cdot \nabla \bar{\sigma}^{c}(\boldsymbol{r})+O\left(\boldsymbol{r}^{\prime}-\boldsymbol{r}\right)^{2}\right] \cdot \boldsymbol{n}_{d} d \boldsymbol{S}^{\prime} \\
& \quad-\frac{1}{\mathscr{V}} \int_{C V S d} \bar{\sigma}^{c} \cdot \boldsymbol{n}_{s} d \boldsymbol{S}^{\prime} .
\end{aligned}
$$


The first term in the Taylor series integrates to zero because the surface is closed. The second is determined using the following identity (easily proved using Gauss' theorem):

$$
\int_{S} r n d S=\mathscr{V}_{S} I
$$

where $\mathscr{V}_{S}$ is the volume inside $S$. Thus

$$
\frac{1}{\mathscr{V}} \int_{\substack{\Sigma S_{p}+\Sigma S_{C_{p}} \\+C V S_{d}}}\left(\boldsymbol{r}^{\prime}-\boldsymbol{r}\right) \cdot \nabla \bar{\sigma}^{c}(\boldsymbol{r}) \cdot \boldsymbol{n}_{d} d \boldsymbol{S}^{\prime}=\epsilon_{d} \nabla \cdot \bar{\sigma}^{c} .
$$

Terms $O\left(\left|\boldsymbol{r}^{\prime}-\boldsymbol{r}\right|^{2}\right)$ are neglected and the last integral in (38) is determined using (34). Thus

$$
\frac{1}{\mathscr{V}} \int_{\Sigma S_{p}+\Sigma S_{C_{p}}} \bar{\sigma}^{c} \cdot \boldsymbol{n}_{d} d \boldsymbol{S}^{\prime} \approx \epsilon_{d} \nabla \cdot \bar{\sigma}^{c}-\nabla \cdot\left(\epsilon_{d} \bar{\sigma}^{c}\right) \approx-\bar{\sigma}^{c} \cdot \nabla \epsilon_{d},
$$

and using (36), (37) and (41), equation (35) becomes

$$
\overline{\left(\frac{1}{\rho} \nabla \cdot \sigma\right)}=\left(\frac{\epsilon_{d}}{\rho_{d}}+\frac{\epsilon_{c}}{\rho_{c}}\right) \nabla \cdot \bar{\sigma}-\frac{1}{\mathscr{V}} \frac{\delta \rho}{\rho_{d} \rho_{c}} \int_{\Sigma S_{p}+\Sigma S_{c_{p}}} \sigma_{c}^{\prime \prime} \cdot \boldsymbol{n}_{d} d \boldsymbol{S}^{\prime}
$$

The fluctuating part of the stress, $\sigma_{c}^{\prime \prime}$ is associated with the stress that arises as a result of the relative motion between the droplets and fluid. The integral of $\sigma_{c}^{\prime \prime}$ over a droplet surface is therefore the force that arises due to this relative motion, that is, it is the drag on the fluid from the droplet. Because flow on the scale of a droplet is assumed to be laminar and because $\epsilon_{d} \ll 1$, droplet-droplet interactions are neglected and each droplet is considered in isolation. As a first approximation for the drag force (which will be modified below), Hadamard [11] drag is assumed. The force on one isolated spherical droplet of radius $a$ is thus

$$
f_{p}=-6 \pi \mu_{c} a \frac{2 \mu_{c}+3 \mu_{d}}{3 \mu_{c}+3 \mu_{d}} V_{r} .
$$

In the limit $\mu_{d} \rightarrow \infty$, the droplet becomes a solid particle and the force reduces to Stokes drag, that is $f_{p}=-6 \pi \mu_{c} a V_{r}$. The difference between the particle velocity and the fluid velocity is approximated by the average relative velocity between phases, that is, $V_{r} \approx U_{R}$. Thus

$$
\frac{1}{\mathscr{V}} \int_{\Sigma S_{p}} \sigma_{c}^{\prime \prime} \cdot n_{c} d S^{\prime}=\frac{1}{\mathscr{V}} N 6 \pi \mu_{c} a U_{R}
$$


where $N$ is the number of uncut particles in the control volume.

The drag term resulting from the integral of $\sigma^{c}$ over $\Sigma S_{C_{p}}$ (that portion of cut particle surfaces lying inside $\boldsymbol{C V}$ ) is more difficult to analyse and the assumption is made that the sum of such contributions from all such particles is equal to the 'drag per unit volume' for one particle multiplied by the sum of volume contributions from the cut particles, that is,

$$
\int_{\Sigma S_{C_{p}}} \sigma_{c}^{\prime \prime} \cdot n_{c} d S^{\prime}=\Sigma V_{C_{p}}\left(\frac{6 \pi \mu_{c} a U_{R}}{\frac{4}{3} \pi a^{3}}\right) .
$$

Because the void fraction is

$$
\epsilon_{d}=\frac{1}{\mathscr{V}}\left(N \frac{4}{3} \pi a^{3}+\Sigma V_{C_{p}}\right)
$$

equations (43) and (44) sum to give the total drag

$$
\frac{1}{\mathscr{V}} \frac{\delta \rho}{\rho_{d} \rho_{c}} \int_{\Sigma S_{p}+\Sigma S_{\mathcal{C}_{p}}} \sigma_{c}^{\prime \prime} \cdot \boldsymbol{n}_{c} d \boldsymbol{S}^{\prime}=\frac{\delta \rho}{\rho_{d} \rho_{c}} \epsilon_{d} K \boldsymbol{U}_{R}
$$

where for a dilute system $K=(9 \mu) /\left(2 a^{2}\right)$. Finally, substituting (46) into (42) yields

$$
\overline{\left(\frac{1}{\rho} \nabla \cdot \sigma\right)}=\left(\frac{\epsilon_{d}}{\rho_{d}}+\frac{\epsilon_{c}}{\rho_{c}}\right) \nabla \cdot \bar{\sigma}+\frac{\delta \rho}{\rho_{d} \rho_{c}} \epsilon_{d} K \boldsymbol{U}_{R}
$$

and the equation for $\bar{U}$ becomes

$$
\frac{\partial \bar{U}}{\partial t}+\nabla \cdot\left(\bar{U} \bar{U}+\epsilon_{d} \epsilon_{c} U_{R} U_{R}\right)=\boldsymbol{g}+\left(\frac{\epsilon_{d}}{\rho_{d}}+\frac{\epsilon_{c}}{\rho_{c}}\right) \nabla \cdot \bar{\sigma}+\frac{\delta \rho}{\rho_{d} \rho_{c}} \epsilon_{d} K \boldsymbol{U}_{R} .
$$

As expected, a drag term appears in this equation when $\rho_{d} \neq \rho_{c}$. When the densities are equal, the only way in which the dispersed phase affects the flow here is by increasing the effective viscosity of the mixture. Also, the averaged momentum equation and equation for mixture-averaged velocity become identical (that is, $\bar{U}=\widetilde{U}$ ) and because drag cannot appear in the momentum equation, it cannot appear in the equation for $\bar{U}$.

\section{The two-field mass and momentum equations}

Transport equations for void-fraction follow from (20) and (21) and using $\epsilon_{d}+\epsilon_{c}=1$

$$
\frac{\partial \epsilon_{m}}{\partial t}+\nabla \cdot\left(\epsilon_{m} \bar{U}^{m}\right)=0 \quad(m=d, c)
$$


Algebraic manipulation of equations (11), (20), (24) and (48) give

$$
\rho_{d} \frac{\partial}{\partial t}\left(\epsilon_{d} \overline{\boldsymbol{U}}^{d}\right)+\rho_{d} \nabla \cdot\left(\epsilon_{d} \overline{\boldsymbol{U}}^{d} \overline{\boldsymbol{U}}^{d}\right)=\rho_{d} \epsilon_{d} \boldsymbol{g}+\epsilon_{d} \nabla \cdot \bar{\sigma}-\epsilon_{d} K \boldsymbol{U}_{R},
$$

and

$$
\rho_{c} \frac{\partial}{\partial t}\left(\epsilon_{c} \overline{\boldsymbol{U}}^{c}\right)+\rho_{c} \nabla \cdot\left(\epsilon_{c} \overline{\boldsymbol{U}}^{c} \overline{\boldsymbol{U}}^{c}\right)=\rho_{c} \epsilon_{c} \boldsymbol{g}+\epsilon_{c} \nabla \cdot \bar{\sigma}+\epsilon_{d} K \boldsymbol{U}_{R}
$$

As expected, the interfacial stress term, $\epsilon_{d} K \boldsymbol{U}_{R}$, appears in these two equations with opposite signs - the momentum lost to one phase due to its interaction with the other appears as a source term in the other equation. Hence momentum of the system as a whole is conserved. The one significant difference between the two-field equations derived here ((50) and (51)) and those often seen in the literature lies in the stress term. For example, under the assumptions of incompressible flow with constant phase density and neglecting turbulence, the equations presented in [5] are equivalent to

$$
\begin{array}{r}
\rho_{d} \frac{\partial}{\partial t}\left(\epsilon_{d} \overline{\boldsymbol{U}}^{d}\right)+\rho_{d} \nabla \cdot\left(\epsilon_{d} \overline{\boldsymbol{U}}^{d} \overline{\boldsymbol{U}}^{d}\right)=\epsilon_{d} \rho_{d} \boldsymbol{g}+\nabla \cdot\left(\epsilon_{d} \bar{\sigma}^{d}\right)+\boldsymbol{F}_{d}, \\
\rho_{c} \frac{\partial}{\partial t}\left(\epsilon_{c} \overline{\boldsymbol{U}}^{c}\right)+\rho_{c} \nabla \cdot\left(\epsilon_{c} \overline{\boldsymbol{U}}^{c} \overline{\boldsymbol{U}}^{c}\right)=\epsilon_{c} \rho_{c} \boldsymbol{g}+\nabla \cdot\left(\epsilon_{c} \bar{\sigma}^{c}\right)+\boldsymbol{F}_{c},
\end{array}
$$

where $\boldsymbol{F}_{d}$ and $\boldsymbol{F}_{c}$ are the interphase forces that are yet to be specified. In [5] the stress terms in (52) and (53) are written

$$
\nabla \cdot\left(\epsilon_{m} \bar{\sigma}^{m}\right)=-\nabla\left(\epsilon_{m} \bar{P}^{m}\right)+\nabla \cdot\left(\epsilon_{m} \mu_{m}\left[\left(\nabla \bar{U}^{m}\right)+\left(\nabla \bar{U}^{m}\right)^{T}\right]\right) \quad(m=c, d) .
$$

As they stand, the stress terms cannot be correct because the void fractions appear inside the gradient of the stress terms. Thus, forces are introduced in the twofield momentum equations from the stress terms purely as a result of dispersedphase concentration gradients. This problem is ameliorated in [5] by postulating an interphase pressure force

$$
\boldsymbol{F}_{d}=\overline{\boldsymbol{P}}^{c} \nabla \epsilon_{d},
$$

which gives the correct pressure force if $\bar{P}^{d}=\bar{P}^{c}$. However, no similar interphase viscous stress force is suggested, and in a subsequent paper is specifically ruled out [3]. Similar differences are observed in the momentum transport equations derived here and other previous work ([10], [13]). In contrast to a typical two-field derivation, the one-field approach gives the correct form of the stress terms automatically. 


\section{Relative velocity}

An equation for $U_{R}$ can be derived from equations (49), (50) and (51):

$$
\left(\frac{d \overline{\boldsymbol{U}}^{d}}{d t}-\frac{d \overline{\boldsymbol{U}}^{c}}{d t}\right)=-\frac{\delta \rho}{\rho_{d} \rho_{c}} \nabla \cdot \bar{\sigma}-\frac{\bar{\rho}}{\epsilon_{c} \rho_{d} \rho_{c}} K \boldsymbol{U}_{R} .
$$

The left-hand side of (54) is proportional to (and of the same order as) the added mass force that can be neglected when particle/liquid interpenetration can be modelled using Stokes drag (as is assumed here). The resulting equation for $\boldsymbol{U}_{R}$ is a quasisteady balance between viscous, pressure and body forces and is rewritten

$$
U_{R}=-\frac{\epsilon_{c} \delta \rho}{\bar{\rho} K} \nabla \cdot \bar{\sigma}
$$

This approximation is known as the drift-flux approximation (for example [19]) and simplifies the numerical solution of the equations considerably.

A surprising result of using the drift-flux approximation is that (55) may be used to remove reference to the drag term in the equation for $\bar{U}$, (48), which becomes

$$
\frac{\partial \bar{U}}{\partial t}+\nabla \cdot\left(\bar{U} \bar{U}+\epsilon_{d} \epsilon_{c} \boldsymbol{U}_{R} \boldsymbol{U}_{R}\right)=\boldsymbol{g}+\left(\frac{1}{\bar{\rho}}\right) \nabla \cdot \bar{\sigma} .
$$

The drag terms considered so far are valid for $\epsilon_{d} \ll 1$. In [2] it is shown that $\epsilon_{d}$ in the drag terms in the two-field equations should be replaced by $\epsilon_{d} \epsilon_{c}$. In addition, if the hindered settling of particles is taken into consideration [1] the coefficient of the drag on one particle $6 \pi \mu_{c} a$ should be replaced by $6 \pi \mu_{c} a \epsilon_{c}\left(1-6.55 \epsilon_{d}\right)^{-1}$. Incorporating these two corrections gives for (55)

$$
\boldsymbol{U}_{R}=-\left(1-6.55 \epsilon_{d}\right) \frac{\delta \rho}{\epsilon_{c} \bar{\rho} K} \nabla \cdot \bar{\sigma}
$$

The equation for mixture-averaged velocity, (56), is unaffected by these changes.

\section{Heat transport}

The following equation describes the transport of heat in the mixture on the microscale:

$$
\frac{\partial \rho I}{\partial t}+\nabla \cdot(\rho I \boldsymbol{U})=\nabla \cdot(k \nabla T)+\sigma \cdot \nabla \boldsymbol{U}+H
$$


where $I$ is the internal heat energy per unit mass and is equal to $C T$. Here $C$ is the specific heat coefficient, $k$ is the coefficient of thermal conductivity and $H$ the heat production per unit volume per unit time.

The diffusive time scale for heat transfer within a particle is $\tau_{H}=a^{2} / \kappa$, where $a$ is the radius of the particle and $\kappa$ (the thermal diffusivity) is related to $k$ by $k=\rho C \kappa$. The dynamic time scale for interpenetration of particle and fluid is $\tau_{D}=\mathscr{L} / \mathscr{U}_{R}$. The ratio of the thermal to interpenetration time scale is

$$
\frac{\tau_{H}}{\tau_{D}}=\frac{a}{\mathscr{L}} \frac{\mathscr{U}_{R} a}{\kappa}=\frac{a}{\mathscr{L}} \operatorname{Re}_{I} \operatorname{Pr},
$$

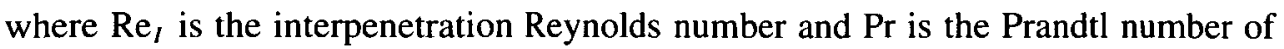
the fluid. $\operatorname{Re}_{I}$ is assumed to be less than 1 (that is, Stokes drag is applicable) and $a \ll \mathscr{L}$. The Prandtl number of fluids such as water is of order 7 and from (59) it is seen that time taken for heat to diffuse through a particle is considerably less than the time taken for a particle to move a significant distance with respect to the fluid. Thus, it is now assumed that the average temperature of the dispersed phase is equal to the average temperature of the continuous phase, that is, $\bar{T}^{d}=\bar{T}^{c}$, or $T_{R}=0$. The specific heat $C$ is also taken to be constant for each material.

When $\bar{T}^{d}=\bar{T}^{c}(=\bar{T})$, the time derivative of (58), when averaged, is

$$
\frac{\partial \overline{\rho I}}{\partial t}=\frac{\partial}{\partial t}\left(\left(\epsilon_{d} \rho_{d} C_{d}+\epsilon_{c} \rho_{c} C_{c}\right) \bar{T}\right) \text {. }
$$

Using (4), (7) and (9), the averaged advection terms can be written

$$
\nabla \cdot(\overline{\rho U I})=\nabla \cdot\left(\left[\epsilon_{d} \rho_{d} C_{d}+\epsilon_{c} \rho_{c} C_{c}\right] \overline{\boldsymbol{U}} \bar{T}+\epsilon_{d} \epsilon_{c}\left[\rho_{d} C_{d}-\rho_{c} C_{c}\right] \boldsymbol{U}_{R} \bar{T}\right),
$$

where the turbulent correlations ${\overline{U^{\prime \prime}}}^{\prime \prime}$ ( $\left.m=d, c\right)$ are neglected.

The average of the diffusive term is written using (1) and (4),

$$
(\overline{k \nabla T})=\frac{1}{\mathscr{V}} \int_{C V}\left(\beta_{d} k_{d}+\beta_{c} k_{c}\right) \nabla\left(\beta_{d} T_{d}+\beta_{c} T_{c}\right) d V^{\prime} .
$$

Taking the coefficient of thermal conductivity inside the gradient, noting that both $k_{d}$ and $k_{c}$ are constant and using (3), gives

$$
(\overline{k \nabla T})=\frac{1}{\mathscr{V}} \int_{C V} \nabla\left(\beta_{d} k_{d} T_{d}+\beta_{c} k_{c} T_{c}\right) d V^{\prime}+k_{R} \frac{1}{\mathscr{V} S_{p}+\Sigma S_{C_{p}}} \int_{n_{d}} d \boldsymbol{S}^{\prime}
$$

Using (34), the first integral in (62) becomes

$$
\frac{1}{\mathscr{V}} \int_{C V} \nabla\left(\beta_{d} k_{d} T_{d}+\beta_{c} k_{c} T_{c}\right) d V^{\prime}=k_{d} \nabla\left(\epsilon_{d} \bar{T}^{d}\right)+k_{c} \nabla\left(\epsilon_{c} \bar{T}^{c}\right) .
$$


The second integral in (62) is manipulated using the decomposition (12) for temperature to give

$$
k_{R} \frac{1}{\mathscr{V}} \int_{\Sigma S_{p}+\Sigma S_{C_{p}}} T n_{d} d \boldsymbol{S}^{\prime}=k_{R} \frac{1}{\mathscr{V}} \int_{\Sigma S_{p}+\Sigma S_{C_{p}}} \bar{T} \boldsymbol{n}_{d} d \boldsymbol{S}^{\prime}+k_{R} \frac{1}{\mathscr{\mathcal { V }}} \int_{\Sigma S_{p}+\Sigma S_{C_{p}}} T^{*} \boldsymbol{n}_{d} d \boldsymbol{S}^{\prime} .
$$

Changing the direction of the surface normal, adding and subtracting an integral over $C V S_{c}$ and expanding in a Taylor series about the control volume centre yields, for the first integral in (64),

$$
\begin{aligned}
k_{R} \frac{1}{\mathscr{V}} & \int_{\Sigma S_{p}+\Sigma S_{C_{p}}} \bar{T} \boldsymbol{n}_{d} d \boldsymbol{S}^{\prime} \\
= & -k_{R} \frac{1}{\mathscr{V}} \int_{\substack{\Sigma S_{p}+\Sigma S_{C} \\
+C V S_{c}}}\left[\bar{T}(\boldsymbol{r})+\left(\boldsymbol{r}^{\prime}-\boldsymbol{r}\right) \cdot \nabla \bar{T}(\boldsymbol{r})+O\left(\boldsymbol{r}^{\prime}-\boldsymbol{r}\right)^{2}\right] \boldsymbol{n}_{c} d \boldsymbol{S}^{\prime} \\
& +k_{R} \frac{1}{\mathscr{V}} \int_{C V S_{c}} \bar{T} \boldsymbol{n}_{s} d \boldsymbol{S}^{\prime},
\end{aligned}
$$

where the normals $\boldsymbol{n}_{c}$ and $\boldsymbol{n}_{s}$ are identical on $C V S_{c}$. The first term in the first integral of (65) is zero. The second term in the first integral is calculated using (39) and terms of $O\left(r^{\prime}-r\right)^{2}$ are ignored. The second integral is calculated using (34). Hence

$$
\begin{aligned}
k_{R} \frac{1}{\mathscr{V}} \int_{\Sigma S_{p}+\Sigma S_{C_{p}}} \bar{T} n_{d} d \boldsymbol{S}^{\prime} & \approx 0-k_{R} \epsilon_{c} \nabla \bar{T}+k_{R} \nabla\left(\epsilon_{d} \bar{T}\right) \\
& =k_{R} \bar{T} \nabla \epsilon_{c} .
\end{aligned}
$$

Substituting (66) into (64), and equations (63) and (64) into (62) yields

$$
\overline{k \nabla T}=\bar{k} \nabla \bar{T}+k_{R} \frac{1}{\mathscr{V}} \int_{\Sigma S_{p}+\Sigma S_{C_{p}}} T^{*} n_{d} d S^{\prime}
$$

Even when the dispersed phase is locally at the same temperature as the fluid, if $k_{R} \neq 0$ then the temperature field is different to the field that would exist in the absence of the dispersed phase. This difference arises from the condition of continuity of heat flux, $k \nabla T$, at the interface between phases. The surface integral of $T^{*}$ represents the change in heat flux due to this difference in the temperature field and cannot be written immediately in terms of averaged variables. It is approximated in the appendix

$$
k_{R} \frac{1}{\mathscr{V}} \int_{\Sigma S_{p}+\Sigma S_{c_{p}}} T^{*} n_{d} d S^{\prime} \approx-\epsilon_{d} \frac{k_{R}^{2}}{\left(k_{d}+2 k_{c}\right)} \nabla \bar{T}
$$


Finally, (67) and (68) combine to give

$$
\overline{k \nabla T}=k_{E} \nabla \bar{T}, \quad \text { where } \quad k_{E}=k_{c}\left(1+\frac{3 \epsilon_{d} k_{R}}{k_{d}+2 k_{c}}\right) .
$$

Viscous dissipation may be neglected in many heat transport problems, and is not considered in the example discussed below. Although the derivation of the average viscous dissipation is not presented here (see [15]) the final result is quoted:

$$
\overline{\sigma: \nabla \boldsymbol{U}}=\bar{\sigma}: \nabla \overline{\boldsymbol{U}}+\frac{\epsilon_{d} \epsilon_{c}^{2}}{\left(1-6.55 \epsilon_{d}\right)} K \boldsymbol{U}_{R} U_{R} .
$$

Finally the average heat transport equation is

$$
\begin{aligned}
\frac{\partial}{\partial t}\left(\left(\epsilon_{d} \rho_{d} C_{d}+\epsilon_{c} \rho_{c} C_{c}\right) \bar{T}\right) & \\
& +\nabla \cdot\left(\left\{\left(\epsilon_{d} \rho_{d} C_{d}+\epsilon_{c} \rho_{c} C_{c}\right) \bar{U}+\epsilon_{d} \epsilon_{c}\left(\rho_{d} C_{d}-\rho_{c} C_{c}\right) \boldsymbol{U}_{R}\right\} \bar{T}\right) \\
= & \nabla \cdot\left(k_{E} \nabla \bar{T}\right)+\bar{\sigma}: \nabla \overline{\boldsymbol{U}}+\frac{\epsilon_{d} \epsilon_{c}^{2}}{\left(1-6.55 \epsilon_{d}\right)} K \boldsymbol{U}_{R} \boldsymbol{U}_{R}+\bar{H}
\end{aligned}
$$

\section{A numerical example}

An example of how the equations derived above can be used to find the solution of a two-phase natural convection is now presented. Details of the appropriate equation scaling and numerical method can be found in [15] and [16] and are not repeated here. The scaled equations are written

$$
\begin{gathered}
\nabla \cdot \overline{\boldsymbol{U}}=0 \\
\frac{\partial \bar{\rho}}{\partial t}+\nabla \cdot\left(\bar{\rho} \overline{\boldsymbol{U}}+\epsilon_{d} \epsilon_{c} \delta \rho \boldsymbol{U}_{R}\right)=0 \\
\operatorname{Re}\left\{\bar{\rho} \frac{\partial \bar{U}}{\partial t}+\bar{\rho} \nabla \cdot\left(\bar{U} \overline{\boldsymbol{U}}+\epsilon_{d} \epsilon_{c} U_{R} \boldsymbol{U}_{R}\right)\right\}=\frac{\operatorname{Ra}}{\mathrm{Pe}\left(\epsilon_{d} \mathscr{F}-\bar{\rho} \bar{T}\right) \hat{\boldsymbol{g}}-\nabla \bar{P}+\nabla \cdot \bar{\tau}} \\
\boldsymbol{U}_{R}=S V_{C} \frac{\mathrm{Pe}}{\operatorname{RaGa}} \frac{f\left(\epsilon_{d}\right)}{\bar{\rho}}\left(\nabla \bar{P}+\rho_{c} \boldsymbol{g}-\nabla \cdot \bar{\tau}\right) \\
\frac{\partial \bar{\rho} \bar{T}}{\partial t}+\nabla \cdot\left(\left[\bar{\rho} \bar{U}+\epsilon_{d} \epsilon_{c} \delta \rho \boldsymbol{U}_{R}\right] \bar{T}\right)=\frac{1}{P e} \nabla^{2} \bar{T}
\end{gathered}
$$


Here $\mathrm{Re}, \mathrm{Ra}, \mathrm{Pe}, \mathrm{Ga}$ are the Reynolds, Rayleigh, Péclet and Gay-Lussac numbers and $\mathscr{F}$ is a parameter that measures the importance of buoyancy due to compositional differences. It is given by

$$
\mathscr{F}=\mathrm{Ga}\left(\frac{\rho_{d}-\rho_{c}}{\rho_{c}}\right) .
$$

The average viscous stress is modelled using

$$
\bar{\tau}=\mu\left(1-\frac{\epsilon_{d}}{0.62}\right)^{-1.55}\left[(\nabla \bar{U})+(\nabla \bar{U})^{T}\right],
$$

where $\mu$ is the liquid viscosity and the multiplying factor is an empirically derived function that is a good fit to the experimental data of [18]. The parameter $S$ is the ratio of particle settling velocity to maximum vertical convection velocity $V_{C}\left(V_{C}\right.$ must be estimated at the simulation start) and $f\left(\epsilon_{d}\right)$ is an empirical function that ensures void fractions do not exceed the close packing limit of 0.6. It has further been assumed that the specific heats of the particles and liquid are equal, heating due to viscous dissipation is negligible and there are no internal heat sources.
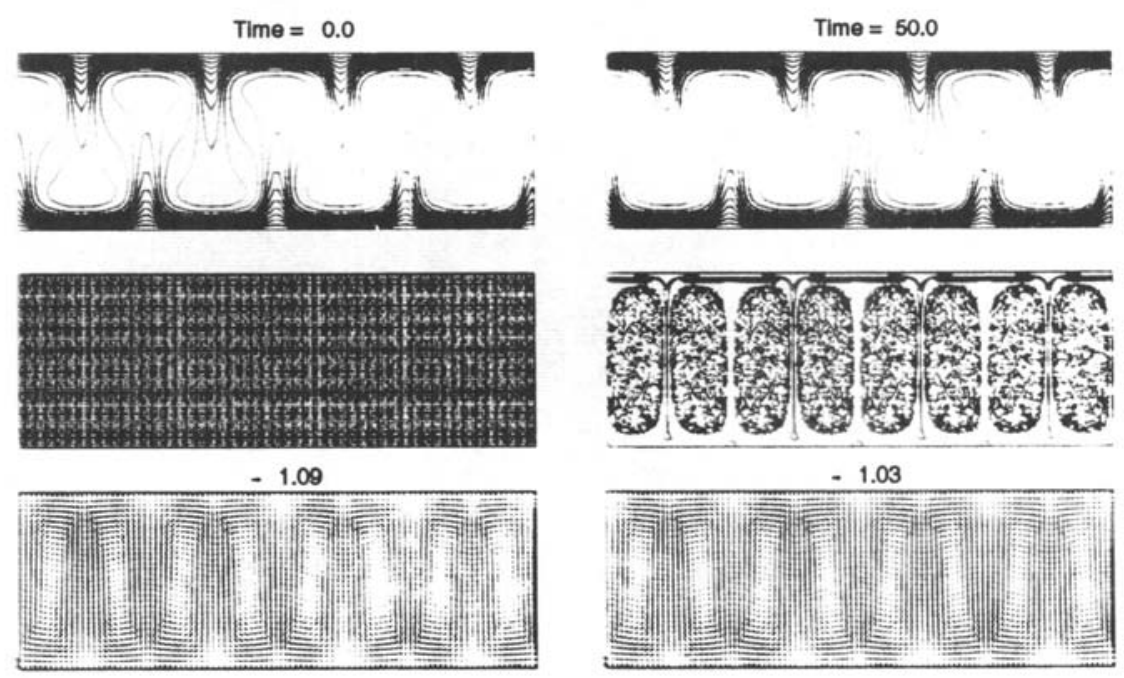

FIGURE 2. Temperature contours (top), particle positions and contours of $\epsilon_{d}$ (middle) and liquid velocity field (bottom) for (a) initial conditions, (b) time $=50$, when $\mathscr{F}=0.1$.

Numerical solution is accomplished using a finite-difference method described in detail in [15]. The computational domain is a regular 2-D Cartesian mesh of size 
$105 \times 35$ and physical dimension of $3 \times 1$, and represents a section of a sill-like magma intrusion of high aspect ratio. Velocity boundary conditions are no-slip at roof and base and free-slip at the side walls. Thermal boundary conditions are heated base $(\bar{T}=1.0)$ and cooled roof $(\bar{T}=0.0)$ and zero normal gradient at the side walls. The initial conditions are shown in Figure $2 \mathrm{a}$, and consist of a vigorous eight-cell convection pattern with $\mathrm{Ra}=10^{5}, \mathrm{Re}=10$ and $\mathrm{Pe}=100$. The liquid has been seeded with a uniform dispersion of particulate matter (with void fraction $\epsilon_{d}=0.05$ and density 1.1 times that of the liquid). At the initial instant, the particles are allowed to move with respect to the fluid, with a nominal settling velocity equal to 0.02 times the maximum vertical convective velocity (equal to 1.0 for the conditions here). The results for $\mathscr{F}=0.1$ after a dimensionless time of 50 are shown in Figure $2 \mathrm{~b}$. There is some initial particle settling, but the majority of the dispersed phase is trapped inside the cores of the convection cells and the flow rapidly reaches a steady state. No further particle settling can occur in this situation.

In contrast to this situation is the case when $\mathscr{F}=5$. Four snapshots of this flow are shown in Figure 3.

In Figure 3a, a similar flow pattern has been established to that observed in Figure $2 b$ for $\mathscr{F}=0.1$. The compositional buoyancy of flow regions where particles have settled are 50 times greater when $\mathscr{F}=5$, and in conjunction with the thermal buoyancy at the base of the cell these regions form unstable buoyant pockets. The flow cannot maintain its steady pattern in this case and the instability that develops causes oscillations in the steady pattern (Figure $3 b$ ) and eventually one of the pockets is able to rise against the down-welling convection cell (Figure 3c). The flow that then arises is very unsteady, and results in the destruction of the original convection pattern with a four-cell pattern establishing itself by the time shown in Figure $3 \mathrm{~d}$. The flow continues to be unsteady until the end of the simulation (time $=50$, not shown).

The instability that arises from the interaction of thermal convection and gravitational settling is important for several reasons. First, it has implications for the crystal settling mechanism that has been proposed as one possible mechanism for the formation of layered igneous intrusions (see [15] and the references therein). During the unsteady cell overturns observed in Figure 3, convection velocities decrease, and substantial amounts of dispersed phase are able to settle. Because the cell pattern is unsteady, there has also been a more uniform dispersal of solids on the base of the cavity than that occurring in Figure $2 \mathrm{~b}$ where the moderate settling occurs in small piles between each pair of cells. The unsteadiness seen in Figure 3 may also be the cause of global instability and subsequent particle re-entrainment that is sometimes observed in minerals processing equipment used to separate solids and liquids at temperatures well above ambient. This connection is currently under investigation, and may ultimately suggest strategies for controlling the problem. 

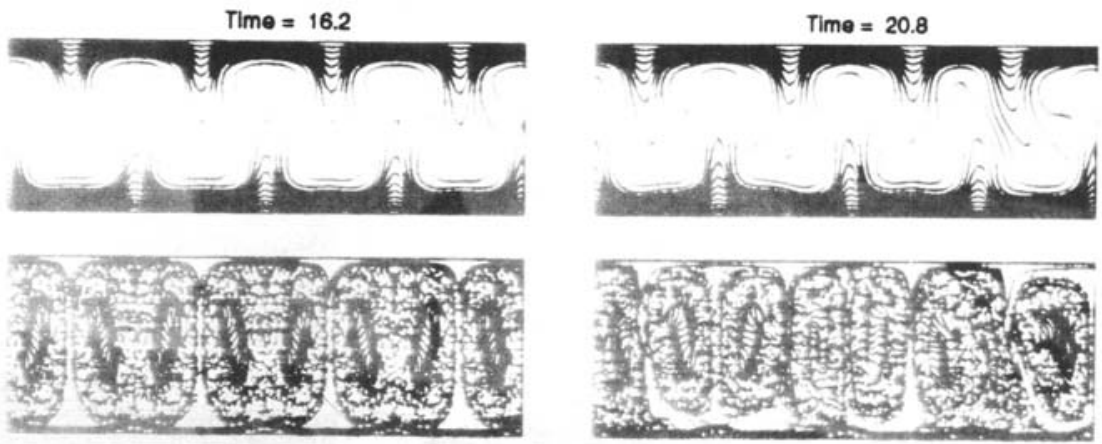

$-0.85$

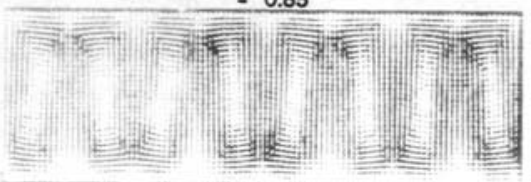

(a)

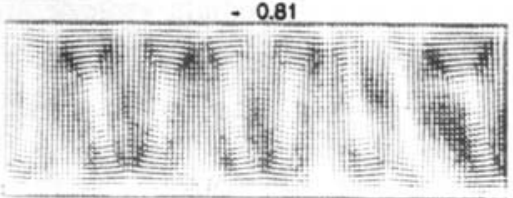

(b)
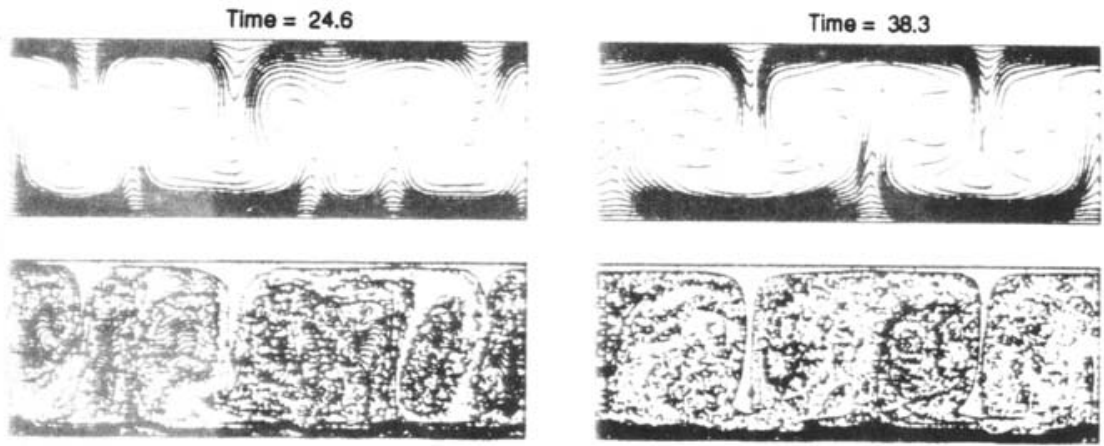

$-0.82$

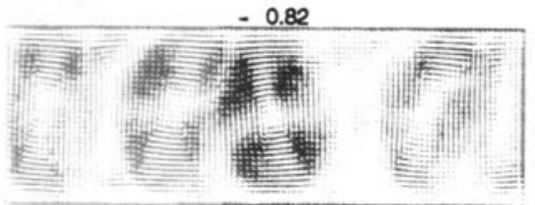

(c)

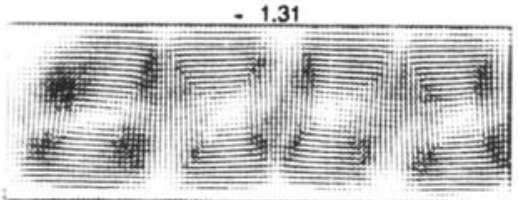

(d)

FIGURE 3. Temperature contours, particle positions and contours of $\epsilon_{d}$ and liquid velocity field for four different times in the unsteady convection that occurs when $\mathscr{F}=5.0$.

\section{Concluding remarks}

A methodology has been proposed to derive the averaged transport equations for mass, momentum and heat in two-phase systems. The approach differs from those 
usually seen in the literature in that all equations are derived as mixture or onefield equations, even though the existence of two distinct phases is acknowledged and utilised heavily in the derivations. Although the one-field approach relies on considerable approximation and on empiricism in deriving constitutive relationships, the situation is no more approximate or empirical than standard two-field approaches. One advantage of using one-field equations is that the formal (albeit approximate) manipulations often lead to physical insight. For example, the void fractions in the pressure and viscous stress terms of two-field momentum equations derived from the one-field equations appear naturally outside of the gradient operator as they should there should be no interpenetration solely because of concentration gradients (at least under the flow conditions and physics considered here). In addition, interaction terms arising at the interface between phases appear naturally because the derivative of the phase function (equation 3 ) locates the interface automatically. Although interaction terms appear as a result of the formalism that is adopted, the exact forms of the interaction must be postulated using physical arguments.

\section{Acknowledgements}

The author thanks Prof J.J. Monaghan of Monash University and Dr F.H. Harlow of the Los Alamos National Laboratory for supervision and encouragement during the course of his Ph.D. degree. Some of this work formed part of the author's Ph.D. thesis undertaken at Monash University and was supported by a Commonwealth Postgraduate Research Award.

\section{Appendix. Average heat diffusion}

To approximate the surface integral in (67), consider an isolated particle of radius $a$ with its centre at the origin. The thermal conductivity of the particle is $k_{d}$ and that of the fluid is $k_{c}$. A temperature gradient exists in the $z$-direction that is a constant, $G$, as $z \rightarrow \pm \infty$. The steady-state temperature field is easily shown to be

$$
\begin{aligned}
& T_{d}=T_{\text {ref }}+\frac{3 k_{c}}{\left(k_{d}+2 k_{c}\right)} G r \cos \theta, \\
& T_{c}=T_{\text {ref }}+\left(r+\frac{\left(k_{c}-k_{d}\right)}{\left(k_{d}+2 k_{c}\right)} \frac{a^{3}}{r^{2}}\right) G \cos \theta,
\end{aligned}
$$

where $T_{\text {ref }}$ is the temperature at the particle's centre.

If the void fraction of dispersed phase in the control volume is $\epsilon_{d}$, then as an approximation, each particle may be considered to lie within a sphere of fluid with 
radius $R=a \epsilon_{d}^{-1 / 3}$. It is now assumed that the surface integral of $T^{*}$ in (67) over one particle is representative of the surface integral over all particles in the control volume. The temperature field given by (A2) is used as an approximation to the temperature field in the vicinity of a particle in the two-component mixture. The influence of other particles on the temperature field and the effect of relative motion between the particle and fluid (which occurs here on a time scale greater than the heat diffusion time scale) are ignored.

For the temperature field given by (A2), the average temperature in both the fluid and the particle is approximated using $T_{r e f}+G r \cos \theta$. Therefore, the $T^{*}$ field needed in the surface integral in (67) is that given by (A2) with $\left(T_{r e f}+G r \cos \theta\right)$ subtracted.

Because (A2) is symmetric about the $z$-axis, the $x$ - and $y$-components of the surface integral are zero, and only the $z$-component remains. The $z$-component of the normal to the sphere is $\cos \theta$, thus the surface integral becomes

$$
k_{R} \frac{1}{\mathscr{V}} \int_{\Sigma S_{p}+\Sigma S_{c_{p}}} T^{*} \boldsymbol{n}_{d} d \boldsymbol{S}^{\prime}=k_{R} \frac{1}{\mathscr{V}} \int_{\phi=0}^{2 \pi} \int_{\theta=0}^{\pi}\left(\frac{k_{c}-k_{d}}{\left(k_{d}+2 k_{c}\right)} G a \cos \theta\right) \cos \theta a^{2} \sin \theta d \theta d \phi .
$$

The sphere of radius $R$ centred on the particle centre takes the place of the control volume $\mathscr{V}$ in this modelling approximation. Its volume is $\left(4 \pi a^{3}\right) /\left(3 \epsilon_{d}\right)$. The integral may then be shown to be

$$
-\epsilon_{d} \frac{k_{R}^{2}}{\left(k_{d}+2 k_{c}\right)} G
$$

Associating the average temperature gradient in the mixture with $G$ gives

$$
\underset{\Sigma}{k_{R} \frac{1}{\mathscr{V}} \int_{p}+\Sigma S_{c_{p}}} T^{*} \boldsymbol{n}_{d} d \boldsymbol{S}^{\prime}=\epsilon_{d} k_{R} \frac{3 k_{c}}{\left(k_{d}+2 k_{c}\right)} \nabla \bar{T}
$$

\section{References}

[1] G. K. Batchelor, "Sedimentation in a dilute dispersion of spheres", J. Fluid Mech. 52 (1972) 245-268.

[2] T. L. Cook and F. H. Harlow, "VORT - A Computer Code for Bubbly Two-phase Flow", Los Alamos National Laboratory Report No. LA-10021-MS, (1978).

[3] D. A. Drew "Two-phase flows: Constitutive equations for lift and Brownian motion and some basic flows", Arch. Rat. Mech. Analysis 62 (1976) 149-163.

[4] D. A. Drew, "Mathematical modelling of two-phase flow", Ann. Rev. Fluid Mech. 15 (1983) 261-291.

[5] D. A. Drew and L. A. Segel, "Averaged equations for two-phase flows", Studies in Appl. Math. L (1971) 205-231. 
[6] D. A. Drew and L. A. Segel, "Analysis of fluidized beds and foams using averaged equations", Studies in Appl. Math. L (1971) 233-257.

[7] A. Einstein, "Eine neue Bestimmung der Moleküldimensionen", Annalen der Physik 19, (1908) 289-307.

[8] W. G. Gray and P. C. Y. Lee, "On the theorems for local volume averaging of multiphase systems, Int. J. Multiphase Flow 3 (1977) 333-340.

[9] G. J. Hwang and H. H. Shen, "Modelling the solid phase stress in a fluid-solid mixture", Int. J. Multiphase Flow. 15 (1989) 257-268.

[10] M. Ishii, Thermo-fluid Dynamic Theory of Two-phase Flow (Eyrolles, Paris, 1975).

[11] H. Lamb, Hydrodynamics, Sixth edition, § 337 (Dover, New York, 1945).

[12] T. S. Lundgren, "Slow flow through stationary random beds and suspensions of spheres", J. Fluid Mech. 51 (1972) 273-299.

[13] J. D. Murray, "On the mathematics of fluidization", J. Fluid Mech. 21 (1965) 465-493.

[14] A. Prosperetti and A. V. Jones, "Pressure forces in disperse two-phase flow", Int. J. Multiphase Flow 10 (1984) 425-440.

[15] M. Rudman, "Low Reynolds number two-phase flows", Ph.D. thesis, Monash University, 1990.

[16] M. Rudman, "Two-phase thermal convection: Implications for crystal settling in magma chambers", Phy. Earth Plan. Int. 72 (1992) 153-172.

[17] G. I. Taylor, "The viscosity of a fluid containing small drops of another fluid", Proc. Roy. Soc. London, A, 138 (1932) 41-48.

[18] D. G. Thomas, "Transport characteristics of suspensions: VIII A note on the viscosity of Newtonian suspensions of uniform spherical particles", J. Coll. Sci. 20 (1965) 267-277.

[19] J. R. Travis, F. H. Harlow and A. A. Amsden, "Numerical Calculation of Two-Phase Flows', Los Alamos Scientific Laboratory Report No. LA-5942-MS (1975).

[20] S. Whitaker, "The transport equations for multi-phase systems", Chem. Eng. Sci. 28 (1973) 139147. 